\title{
REABILITAÇÃO VESTIBULAR EM IDOSOS COM PARKINSON
}

\author{
Vestibular rehabilitation in elderly patients with Parkinson
}

\author{
Jackeline Martins-Bassetto ${ }^{(1)}$, Bianca Simone Zeigelboim ${ }^{(2)}$, Ari Leon Jurkiewicz ${ }^{(3)}$, \\ Angela Ribas ${ }^{(4)}$, Marine Raquel Diniz da Rosa ${ }^{(5)}$
}

\section{RESUMO}

Objetivo: verificar a efetividade dos exercícios de reabilitação vestibular (RV) por meio de avaliação pré e pós-aplicação do questionário Dizziness Handicap Inventory (DHI) - adaptação brasileira. Métodos: avaliaram-se oito pacientes (três do sexo feminino e cinco do sexo masculino), na faixa etária de 48 a 71 anos, encaminhados da Associação Paranaense de Parkinson para o Laboratório de Otoneurologia da Universidade Tuiuti do Paraná. Os pacientes foram divididos em dois grupos e submetidos aos seguintes procedimentos: anamnese, avaliação otorrinolaringológica, avaliação vestibular por meio da vectoeletronistagmografia (VENG) e aplicação do questionário DHI - adaptação brasileira pré e pós RV utilizando-se os protocolos de Cawthorne e Cooksey (grupo A) e Herdman (grupo B). Resultados: a) conforme as queixas otoneurológicas referidas na anamnese, observou-se a prevalência da tontura $(100,0 \%)$, tremor $(100,0 \%)$ e desvio de marcha $(75,0 \&) ; b)$ no exame vestibular, todos os pacientes $(100,0 \%)$ apresentaram alteração, sendo a maior freqüência das síndromes vestibulares periféricas deficitárias $(62,5 \%)$; $)$ houve melhora significativa dos aspectos funcional $(p=$ 0,020470 ) e emocional $(p=0,013631$ ) após a realização dos exercícios de RV utilizando-se o protocolo de Cawthorne e Cooksey e do aspecto emocional $(p=0,007316)$ utilizando-se o protocolo de Herdman. Conclusão: comparando-se os dois protocolos utilizados, verificou-se uma melhora significativa dos pacientes do grupo A, submetidos ao protocolo de Cawthorne e Cooksey $(p=0.0231)$.

DESCRITORES: Testes de Função Vestibular; Doença de Parkinson; Eletronistagmografia; Tontura

\section{INTRODUÇÃO}

O processo de envelhecimento manifesta-se por um declínio das funções de diversos órgãos de forma linear em função do tempo, não se conseguindo definir o ponto exato de transição, como nas demais fases. Observa-se que ao final da terceira década evi-

(1) Fonoaudióloga da Empresa de Aparelhos Audiotec; Mestre em Distúrbios da Comunicação pela Universidade Tuiuti do Paraná.

(2) Fonoaudióloga; Coordenadora do Programa de Mestrado e Doutorado em Distúrbios da Comunicação da Universidade Tuiuti do Paraná, Doutora em Ciências dos Distúrbios da Comunicação Humana pela Universidade Federal de São Paulo.

(3) Médico Clínico; Professor Adjunto do Programa de Mestrado e Doutorado em Distúrbios da Comunicação da Universidade Tuiuti do Paraná, Doutor em Anatomia pela Universidade Federal de São Paulo.

(4) Fonoaudióloga; Professora do Curso de Graduação em Fonoaudiologia da Universidade Tuiuti do Paraná; Mestre em Distúrbios da Comunicação pela Universidade Tuiuti do Paraná.

(5) Fonoaudióloga; aluna do Programa de Mestrado em Distúrbios da Comunicação da Universidade Tuiuti do Paraná. denciam-se as alterações anatomofuncionais atribuídas ao envelhecimento. O número de idosos vem crescendo consideravelmente no Brasil devido às melhorias de condições da saúde pública e os avanços da medicina. Atualmente, 15 milhões de pessoas têm mais de 60 anos; o Brasil é considerado o $6^{\circ}$ país no mundo com o maior número de idosos, em torno de 32 milhões, de acordo com a Organização Mundial de Saúde (OMS). Segundo a OMS, em 2025 serão dois bilhões de pessoas com mais de 60 anos em todo o mundo ${ }^{1,2}$.

O limite de idade entre o indivíduo adulto e o idoso é de 65 anos para as nações desenvolvidas e de 60 anos para os países em desenvolvimento, sendo esse critério cronológico adotado na maioria das instituições que atendem ao idoso. As doenças crônicodegenerativas como as doenças de Parkinson (DP) e de Alzheimer passaram a serem mais incidentes, sendo a DP reconhecida como um dos distúrbios neurológicos mais comuns que acomete principalmente o sistema motor ${ }^{3,4}$.

Com o envelhecimento a velocidade de condução dos impulsos nervosos é reduzida, com alterações nos 
neurotransmissores. A falta de dopamina (neurotransmissor que age nos núcleos da base) acarreta o surgimento da $\mathrm{DP}^{5}$. A etiologia da DP é obscura, mas diversos fatores como: genéticos, aterosclerose, acúmulo excessivo de radicais livres de oxigênio, infecções virais, traumatismo craniano, uso de medicamentos antipsicóticos e fatores ambientais, podem ser desencadeantes da doença ${ }^{6}$.

Segundo os autores ${ }^{7}$ há um comprometimento da habilidade do sistema nervoso central no processamento dos sinais vestibulares, visuais e proprioceptivos responsáveis pela manutenção do equilíbrio corporal, com diminuição da capacidade de modificação dos reflexos adaptativos. Sintomas como a tontura e o desequilíbrio fazem parte dessas alterações sensoriais, que também podem acometer outras idades, mas são mais freqüentes após os 65 anos.

Os idosos com idade superior a 75 anos apresentam a tontura como sendo o sintoma mais evidente. Outros sintomas associados podem estar presentes e relacionados a causas etiológicas de origem sensorial, disfunções cerebrovasculares e cardiovasculares, alterações cervicais doenças metabólicas, neurológicas, ósseas, degenerativas e outras ${ }^{8,9}$. Alterações do controle postural na população idosa causam um risco maior de queda e suas conseqüentes seqüelas com elevada mortalidade ${ }^{9-11}$.

As desordens do sistema vestibular podem gerar problemas emocionais, físicos e incapacidade para performance das atividades da vida diária do paciente 12. As principais formas de tratamento para as disfunções vestibulares são medicamentosa, cirúrgica e reabilitação vestibular $(\mathrm{RV})^{13}$.

A RV tem sido evidenciada por agir fisiologicamente sobre o sistema vestibular, sendo um recurso terapêutico que envolve estimulações visuais proprioceptivas e vestibulares, com o intuito de manter o equilíbrio corporal dos pacientes com sintomas vertiginosos ${ }^{14}$. A RV tem uma proposta de atuação baseada em mecanismos centrais de neuroplasticidade conhecidos como adaptação, habituação e substituição para obtenção da compensação vestibular.

Os exercícios de RV visam melhorar a interação vestibulovisual durante a movimentação cefálica, ampliar a estabilidade postural estática e dinâmica nas condições que geram informações sensoriais conflitantes e diminuir a sensibilidade individual à movimentação cefálica ${ }^{13,15}$. A RV pode promover a cura completa em $30 \%$ dos casos e diferentes graus de melhora em $85 \%$ dos indivíduos ${ }^{13}$.

Como a longevidade humana está em contínuo crescimento e o desequilíbrio é uma constante na diminuição da qualidade de vida nos indivíduos com DP, objetivou-se na presente pesquisa verificar a efetividade dos exercícios de reabilitação vestibular por meio de avaliação pré e pós-aplicação do questionário Dizziness Handicap Inventory - adaptação brasileira.

\section{MÉTODOS}

Avaliaram-se oito pacientes sendo três do sexo feminino e cinco do sexo masculino, na faixa etária de 48 a 77 anos, encaminhados da Associação Paranaense de Parkinson (APP) para o Laboratório de Otoneurologia da Universidade Tuiuti do Paraná. Os pacientes foram divididos em dois grupos; no Grupo A, aplicou-se os exercícios de Cawthorne e Cooksey (protocolo I) - Figura 1 e no Grupo B, aplicou-se os exercícios de Herdman (protocolo II) - Figura 2. Não houve critério na divisão dos grupos.

Os pacientes foram avaliados independente do tempo de tratamento. Foram excluídos da pesquisa pacientes que apresentaram comprometimentos psicológicos, visuais, reumatológicos, muscoesqueléticos importantes e outros distúrbios que impossibilitassem a realização da avaliação e reabilitação vestibular.

Os pacientes foram submetidos aos seguintes procedimentos:

\section{Anamnese}

Aplicou-se um questionário (Figura 3) com ênfase aos sinais e sintomas otoneurológicos.

\section{Avaliação Otorrinolaringológica}

Realizada pelo médico no laboratório de otoneurologia com o objetivo de excluir qualquer alteração que pudesse interferir no exame.

\section{Avaliação Vestibular}

Realizada pelas fonoaudiólogas do estudo. Os pacientes foram submetidos às seguintes provas que compõem o exame vestibular:

\section{-Sem registro}

* Pesquisou-se o nistagmo e a vertigem de posição/posicionamento por meio da manobra de Brandt e Daroff ${ }^{16}$;

* Pesquisaram-se os nistagmos espontâneo e semi-espontâneo com os olhos abertos, no olhar frontal e a 30 graus de desvio do olhar para a direita, esquerda, para cima e para baixo.

\section{-Com registro}

Para a realização da VENG utilizou-se um apareIho termossensível, com três canais de registro, da marca Berger, modelo VN316. Após a limpeza da pele 
das regiões periorbitárias com álcool, colocaram-se em cada paciente, fixados com pasta eletrolítica, um eletródio ativo no ângulo lateral de cada olho e na linha média frontal, formando um triângulo isóscele, que permitiu a identificação dos movimentos oculares horizontais, verticais e oblíquos. Este tipo de VENG permitiu obter medidas mais precisas da velocidade angular da componente lenta (correção vestibular) do nistagmo.

Utilizou-se uma cadeira rotatória pendular decrescente da marca Ferrante, de um estimulador visual marca Neurograff, modelo EV VEC, e de um otocalorímetro a ar, da marca Neurograff, modelo NGR 05.

Realizaram-se as seguintes provas oculares e labirínticas à VENG, segundo os critérios propostos por diversos autores ${ }^{17,18}$.

" Calibração dos movimentos oculares: nesta etapa do exame avaliou-se a regularidade do traçado, o que torna as pesquisas comparáveis entre si;

"Pesquisa dos nistagmos espontâneo (olhos abertos e fechados) e semi-espontâneo (olhos abertos): nesse registro avaliaram-se a ocorrência, direção, efeito inibidor da fixação ocular (EIFO) e o valor da velocidade angular da componente lenta (VACL) máxima do nistagmo;

" Pesquisa do rastreio pendular: avaliaram-se a ocorrência e o tipo de curva;

" Pesquisa do nistagmo optocinético, à velocidade de $60^{\circ}$ por segundo, nos sentidos anti-horário e horário, na direção horizontal. Avaliaram-se a ocorrência, direção, VACL máxima às movimentações anti-horária e horária do nistagmo.

" Pesquisa dos nistagmos pré e pós-rotatórios à prova rotatória pendular decrescente, com estimulação dos ductos semicirculares laterais, anteriores e posteriores. Para a estimulação dos ductos semicirculares laterais (horizontais) a cabeça foi fletida $30^{\circ}$ para frente. $\mathrm{Na}$ etapa seguinte, para a sensibilização dos ductos semicirculares anteriores e posteriores (verticais) o posicionamento da cabeça foi de $60^{\circ}$ para trás e $45^{\circ}$ à direita e, a seguir, $60^{\circ}$ para trás e $45^{\circ}$ à esquerda, respectivamente. Observaramse a ocorrência, direção, freqüência às rotações antihorária e horária do nistagmo.

Pesquisa dos nistagmos pré e pós-calóricos realizada com o paciente posicionado de forma que a cabeça e o tronco estivessem inclinados $60^{\circ}$ para trás, para estimulação adequada dos ductos semicirculares laterais. O tempo de irrigação de cada oreIha com ar a $42^{\circ} \mathrm{C}, 20^{\circ} \mathrm{C}$ e $10^{\circ} \mathrm{C}$ durou 80 s para cada temperatura e as respostas foram registradas com os olhos fechados e, a seguir, com os olhos abertos para a observação do EIFO. Nesta avaliação verificaram-se a direção, os valores absolutos da VACL e o cálculo das relações da preponderância direcional e predomínio labiríntico do nistagmo pós-calórico. leira

Aplicação do questionário DHI - adaptação brasi-

Este questionário foi elaborado por Jacobson \& Newman ${ }^{19}$ e adaptado culturalmente à população brasileira por Castro ${ }^{20}$, como mostra a Figura 4 . O questionário foi aplicado pré e pós a aplicação dos exercícios de reabilitação vestibular. Objetivou-se verificar o grau de desvantagem que a tontura causa na prática diária e avaliar os aspectos emocional e funcional com nove questões cada e o aspecto físico, com sete questões, no total de 25 quesitos. As respostas permitidas foram "sim", equivalente a quatro pontos, "às vezes", equivalente a dois pontos e "não", equivalente a zero. A pontuação varia de zero a 100 pontos, sendo que quanto mais próximo de 100 maior será a desvantagem causada pela tontura na vida do paciente.

Protocolos de reabilitação vestibular de Cawthorne ${ }^{21}$ e Cooksey $^{22}$ - Protocolo I e de Herdman ${ }^{23}$ - Protocolo II

Os exercícios foram realizados durante três meses, duas vezes por semana, no Laboratório de Otoneurologia da Universidade Tuiuti do Paraná, sob supervisão de uma fonoaudióloga.

No protocolo I, os exercícios objetivam promover o retorno da função dos equilíbrios estático e dinâmico, com restauração da orientação espacial. Os exercícios são realizados por meio de movimentos dos olhos, cabeça e corpo nas posições sentada e ortostática.

No protocolo II, os exercícios objetivam promover adaptação vestibular, a estabilização das posturas estática e dinâmica do campo visual. Os exercícios são realizados através de movimentos dos olhos e cabeça em pé, além de caminhar.

Estes protocolos foram selecionados por serem de fácil aplicação na população idosa e permitir a realização em grupo, com uma maior interação entre os idosos.

Este estudo foi aprovado pelo Comitê de Ética Institucional parecer $\mathrm{n}^{\circ}$. 008/2005 e autorizado pelos pacientes pela assinatura do Termo de Consentimento Livre e Esclarecido.

Efetuou-se a análise descritiva dos dados da anamnese e da avaliação vestibular. Para análise dos dados do questionário DHI - adaptação brasileira, aplicou-se o teste t de Student unilateral e foi adotado o nível de significância 0,05 ou $5 \%$ para a rejeição de hipótese de nulidade.

\section{RESULTADOS}

Com relação às queixas e sintomas referidos na anamnese, observou-se a freqüência do tremor $(100,0 \%)$, tontura $(100,0 \%)$ e desvio de marcha $(75,0 \%)$ para os pacientes do grupo A e tremor $(100,0 \%)$ e tontura $(100,0 \%)$ para os pacientes do grupo B, como demonstra a Tabela 1. 
Com relação à avaliação da função vestibular, constatou-se alteração em todos os idosos $(100,0 \%)$ com predomínio da hiporreflexia labiríntica unilateral $(37,5 \%)$ no grupo A e bilateral $(25,0 \%)$ no grupo B, seguida da hiperrreflexia labiríntica $(25,0 \%)$ conforme demonstra a Tabela 2.

Evidenciou-se no exame vestibular a freqüência da síndrome vestibular periférica deficitária em ambos os grupos, em cinco pacientes $(62,5 \%)$, conforme mostra a Tabela 3.

$\mathrm{Na}$ análise do questionário $\mathrm{DHI}$ - adaptação brasileira, do grupo A, utilizando-se o protocolo I, observou-se uma melhora na pontuação total com variação de 16 a 44, média de 28, e desvio-padrão de 10,4 no pré-tratamento e de zero a 12 pontos, média de 5,5 , e desvio-padrão de 4,3 no pós-tratamento, como mostra a Tabela 4.

De acordo com o teste t de Student comparandose as avaliações pré e pós a RV, verificou-se resultado significativo nos aspectos funcional e emocional.
$\mathrm{Na}$ análise do questionário $\mathrm{DHI}$ - adaptação brasileira, do grupo B, utilizando-se o protocolo II, observou-se uma melhora significativa na pontuação total com variação de 14 a 76 pontos, média de 34 , e desvio-padrão de 24,6 no pré-tratamento e de zero a 16 pontos, média de 8,5 , e desvio-padrão de 5,7 no póstratamento, como mostra a Tabela 5.

De acordo com o teste t de Student comparandose as avaliações pré e pós a RV, verificou-se resultado significativo no aspecto emocional.

Analisando-se as médias pré e pós-tratamento dos aspectos físico, funcional e emocional, dos grupos A e B em cada protocolo aplicado, observou-se uma diminuição e conseqüente melhora, como mostram as Figuras 5 e 6.

Comparando-se os dos dois protocolos de reabilitação utilizados no pré e pós-tratamento, de acordo com o teste t de Student, observou-se uma melhora significativa dos pacientes do grupo A, submetidos ao protocolo I conforme demonstra a Figura 7.

\begin{tabular}{|c|c|}
\hline \multicolumn{2}{|r|}{ MOVIMENTOS DE OLHOS E CABEÇA NA POSIÇÄO SENTADA } \\
\hline 1. & Olhar para cima e para baixo \\
\hline 2. & Olhar para a direita e para a esquerda \\
\hline 3. & Aproximar e afastar o dedo, olhando para ele (lentamente e depois rapidamente) \\
\hline 4. & Mover a cabeça em flexão e extensão com os olhos abertos (lentamente e depois rapidamente) \\
\hline 5. & Mover a cabeça para a direita e para esquerda com os olhos (lentamente e depois rapidamente) \\
\hline 6. & Repetir os exercícios 4 e 5 com os olhos fechados \\
\hline \multicolumn{2}{|r|}{ MOVIMENTOS DE CABEÇA E CORPO NA POSIÇÃO SENTADA } \\
\hline $\begin{array}{l}1 . \\
2 .\end{array}$ & $\begin{array}{l}\text { Colocar um objeto no chão e apanhá-lo realizando o movimento de flexão e extensão do tronco } \\
\text { (olhar para o objeto o tempo todo) } \\
\text { Flexionar o tronco e passar um objeto pela frente e por trás dos joelhos }\end{array}$ \\
\hline \multicolumn{2}{|r|}{ EXERCÍCIOS NA POSIÇÃO ORTOSTÁTICA } \\
\hline 1. & Sentar e levantar para a posição ortostática com os olhos abertos \\
\hline 2. & Repetir o exercício 1 com os olhos fechados \\
\hline 3. & Repetir o exercicio 1 fazendo, porém uma volta para a direita na posição ortostática \\
\hline 4. & Repetir o exercício 1 fazendo, porém, uma volta para a esquerda na posição ortostática \\
\hline \multicolumn{2}{|r|}{ ATIVIDADE PARA MELHORAR O EQUILÍBRIO } \\
\hline 1. & Caminhar fazendo rotação cervical para a direita e para a esquerda \\
\hline 2. & $\begin{array}{l}\text { Na posição ortostática fazer voltas repentinas de } 90^{\circ} \text { com o corpo (com os olhos abertos e, } \\
\text { depois, com os olhos fechados) }\end{array}$ \\
\hline 3. & Subir e descer escadas (usar o corrimão, se necessário) \\
\hline 4. & $\begin{array}{l}\text { Na posição ortostática, ficar em um pé (com o pé direito e, depois, com o pé } \\
\text { esquerdo), com os olhos abertos e, depois com os olhos fechados }\end{array}$ \\
\hline 5. & Ficar na posição ortostática sobre uma superficie macia \\
\hline 6. & Caminhar sobre uma superficie macia \\
\hline 7. & Andar pé-ante-pé com os olhos abertos e, depois, com os olhos fechados \\
\hline 8. & Repetir o exercício 4 em uma superficie macia. \\
\hline
\end{tabular}

Figura 1 - Exercícios Cawthorne e Cooksey (Protocolo I) 


\section{EXERCÍCIOS PARA AUMENTAR A ESTABILIDADE POSTURAL ESTÁTICA E DINÃMICA}

1. Ficar em pé, com os pés juntos quanto possível para manter o equilíbrio, mesmo que com a ajuda das mãos apoiadas em uma parede. Tire uma e depois as duas mãos progressivamente

2. Ficar em pé, com os pés o mais afastado possível mirando um alvo a sua frente. Estreite progressivamente a base de sustentação colocando sucessivamente os Pés juntos, um pé parcialmente adiante do outro, com os braços abertos, depois ao longo do corpo e por fim cruzados no peito

3. Ficar em pé, com os pés o mais afastado possível, com a cabeça fletida $\mathbf{3 0}$ graus para a frente, com os braços abertos, depois ao longo do corpo e por fim cruzados no peito

4. Ficar em pé, com os pés o mais afastado possível, com a cabeça fletida 30 graus para atrás, com os braços abertos, depois ao longo do corpo e por fim cruzados no peito

5. Realizar os exercícios números 1,2,3 e 4 com os olhos fechados

6. Realizar os exercícios números 1,2,3 e 4 em cima de um travesseiro ou colchonete

7. Andar próximo a uma parede com os olhos abertos, procurando gradualmente estreitar a base, até conseguir andar com um pé adiante do outro

8. Realizar o exercício número 7 com os olhos fechados

9. Andar próximo a uma parede, girando a cabeça para a direita e esquerda, fixando os olhos em objetos no ambiente.

10. Dar cinco passos e girar 180 graus para a direita, posteriormente dar mais cinco passos e virar 180 graus para a esquerda

11. Andar em um supermercado e/ou Shopping Center sem apoio, inicialmente em um horário de menos movimento, e a seguir, em horário de mais movimento

Figura 2 - Exercícios de Herdman (Protocolo II) 


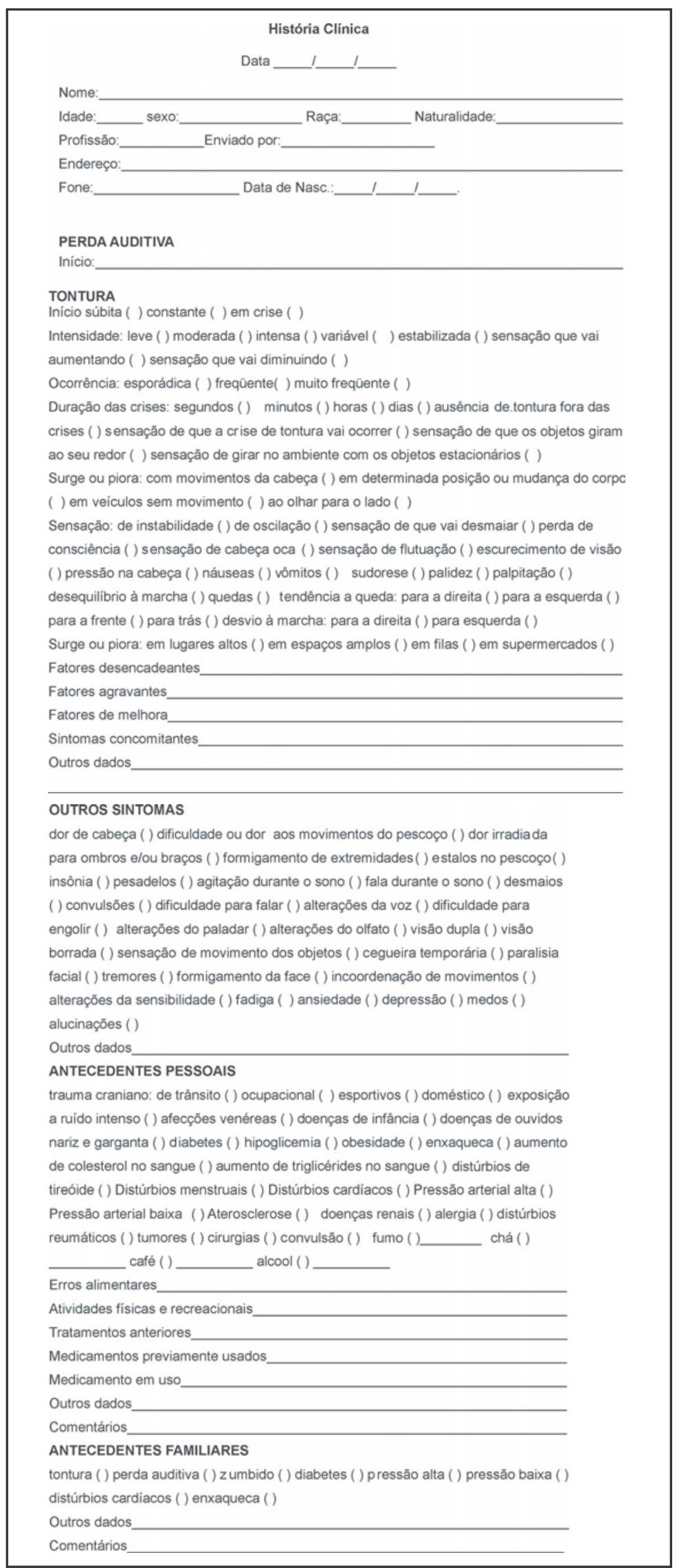

Figura 3 - Questionário utilizado no laboratório de otoneurologia da Universidade Tuiuti do Paraná 


\begin{tabular}{lcc} 
& DHI & \multicolumn{2}{c}{ RESPOSTAS } \\
\hline \multirow{2}{*}{ Aspecto } & SIM ÀS VEZES NÃO & PONTUAÇÃo
\end{tabular}

(4) (2) (0)

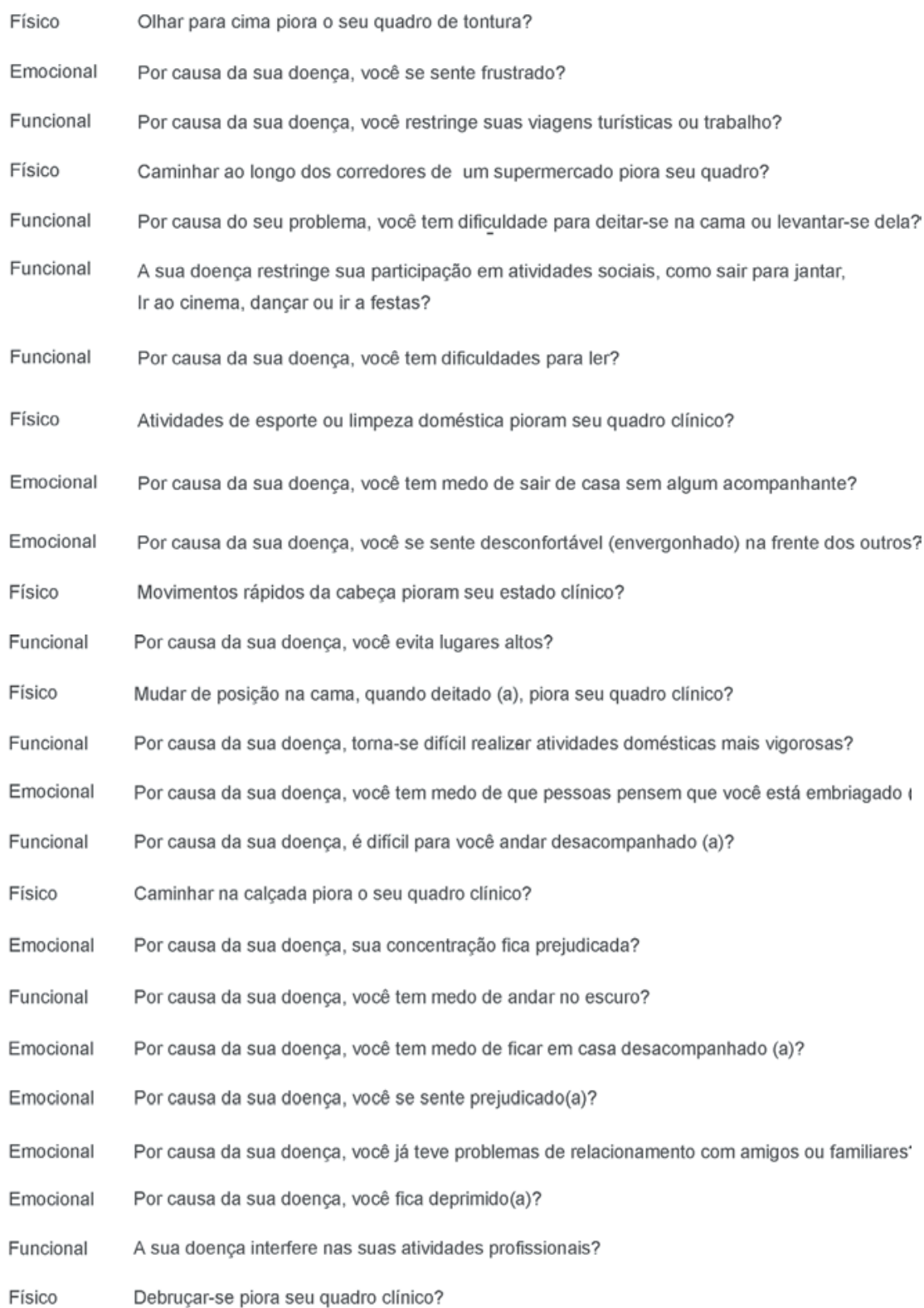

TOTAL: pontos

Figura 4 - Questionário de handicap para tontura 
Tabela 1 - Freqüência das queixas e sintomas apontados na anamnese nos grupos A e B

\begin{tabular}{lcccc}
\hline \multicolumn{1}{c}{ GUEIXAS E SINTOMAS } & N & $\%$ & N & $\%$ \\
\hline Tremor & 4 & 100,0 & 4 & 100,0 \\
Tontura & 4 & 100,0 & 4 & 100,0 \\
Desvio de marcha & 3 & 75,0 & - & - \\
Zumbido & 1 & 25,0 & - & - \\
Queda & 1 & 25,0 & 1 & 25,0 \\
Ansiedade & 1 & 25,0 & - & - \\
Depressão & 1 & 25,0 & - & 25,0 \\
Instabilidade & - & - & 1 & 25,0 \\
Desequilibrio à marcha & - & - & 1 & \\
\hline
\end{tabular}

$\mathrm{N}$ - número de pacientes

Tabela 2 - Freqüência de achados anormais à avaliação vestibular nos grupos A e B

Freqüência

\begin{tabular}{lcc}
\multicolumn{1}{c}{ ACHADOS } & Grupo A & Grupo B \\
\hline Hiporreflexia Labiríntica unilateral & 2 & - \\
Hiporreflexia Labiríntica bilateral & 1 & 2 \\
Hiperreflexia Labiríntica unilateral & 1 & 2 \\
PDN alterada & - & - \\
\hline
\end{tabular}

PDN: Preponderância direcional do nistagmo

Tabela 3 - Resultado do exame vestibular nos grupos A e B

\begin{tabular}{|c|c|c|c|c|}
\hline & \multicolumn{2}{|c|}{ GRUPO A } & \multicolumn{2}{|c|}{ GRUPO B } \\
\hline RESULTADO & $\mathrm{N}$ & $\%$ & $\mathrm{~N}$ & $\%$ \\
\hline S.V.P.D.U. & 2 & 50,0 & - & - \\
\hline S.V.P.I.U & 1 & 25,0 & - & - \\
\hline S.V.P.D.B & 1 & 25,0 & 2 & 50,0 \\
\hline S.V.P.I. & - & - & 2 & 50,0 \\
\hline
\end{tabular}

$\mathrm{N}$ - número de pacientes; S.V.P.D.U. - síndrome vestibular periférica deficitária unilateral; S.V.P.I.U - síndrome vestibular periférica irritativa unilateral; S.V.P.D.B. - síndrome vestibular periférica deficitária bilateral; S.V.P.I. - síndrome vestibular periférica irritativa. 
Tabela 4 - avaliação dos aspectos físico, funcional e emocional pré e pós tratamento do grupo A, utilizando o protocolo I

\begin{tabular}{cccccccccr}
\hline PACIENTE & \multicolumn{2}{c}{ FísICO } & \multicolumn{2}{c}{ FUNCIONAL } & \multicolumn{2}{c}{ EMOCIONAL } & \multicolumn{2}{c}{ TOTAL } \\
\hline & Pré & Pós & Pré & Pós & Pré & Pós & Pré & Pós \\
\hline 1 & 2 & 4 & 6 & - & 8 & - & 16 & 4 \\
2 & 16 & 2 & 20 & 4 & 8 & - & 44 & 6 \\
3 & 6 & 2 & 8 & 4 & 8 & 6 & 22 & 12 \\
4 & 8 & - & 12 & - & 10 & - & 30 & - \\
Média & 8 & 2 & 11,5 & 2 & 8,5 & 1,5 & 28 & 5,5 \\
DP & 5,9 & 1,6 & 6,2 & 2,3 & 1,0 & 3,0 & 10,4 & 4,3 \\
\hline
\end{tabular}

DP - desvio padrão

A aplicação do teste t de Student comparando as avaliações pré e pós RV (Protocolo I) resultou nos valores de $p=0,086362$ (físico); $p=0,020470$ (funcional) e $p=0,013631$ (emocional). Considerando-se o nível de significância de $5 \%(\alpha=0,05)$, verificou-se que o resultado é significativo para os aspectos funcional e emocional.

Tabela 5 - Avaliação dos aspectos físico, funcional e emocional pré e pós tratamento do grupo B, utilizando o protocolo II

\begin{tabular}{crrrrrrrrr}
\hline PACIENTE & \multicolumn{2}{c}{ FÍSICO } & \multicolumn{2}{c}{ FUNCIONAL } & \multicolumn{2}{c}{ EMOCIONAL } & \multicolumn{2}{c}{ TOTAL } \\
\hline & Pré & Pós & Pré & Pós & Pré & Pós & Pré & Pós \\
\hline 1 & - & 4 & 18 & 8 & 8 & 4 & 20 & 16 \\
2 & 14 & 6 & 12 & 2 & - & - & 26 & 8 \\
3 & - & - & 6 & - & 8 & - & 14 & - \\
4 & 20 & 4 & 22 & 6 & 28 & - & 76 & 10 \\
Média & 8,5 & 3,5 & 14,5 & 4 & 11 & 1 & 34 & 8,5 \\
DP & 10,1 & 2,5 & 7,0 & 3,7 & 11,9 & 2 & 24,6 & 5,7 \\
\hline
\end{tabular}

DP - Desvio padrão

A aplicação do teste t de Student comparando as avaliações pré e pós RV (Protocolo II) resultou nos valores de $p=0,170788$ (físico); $p=0,103082$ (funcional) e $p=0,007316$ (emocional), considerando-se o nível de significância de 5\% ( $\alpha=0,05)$, verificou-se que o resultado é significativo apenas para o aspecto emocional. 


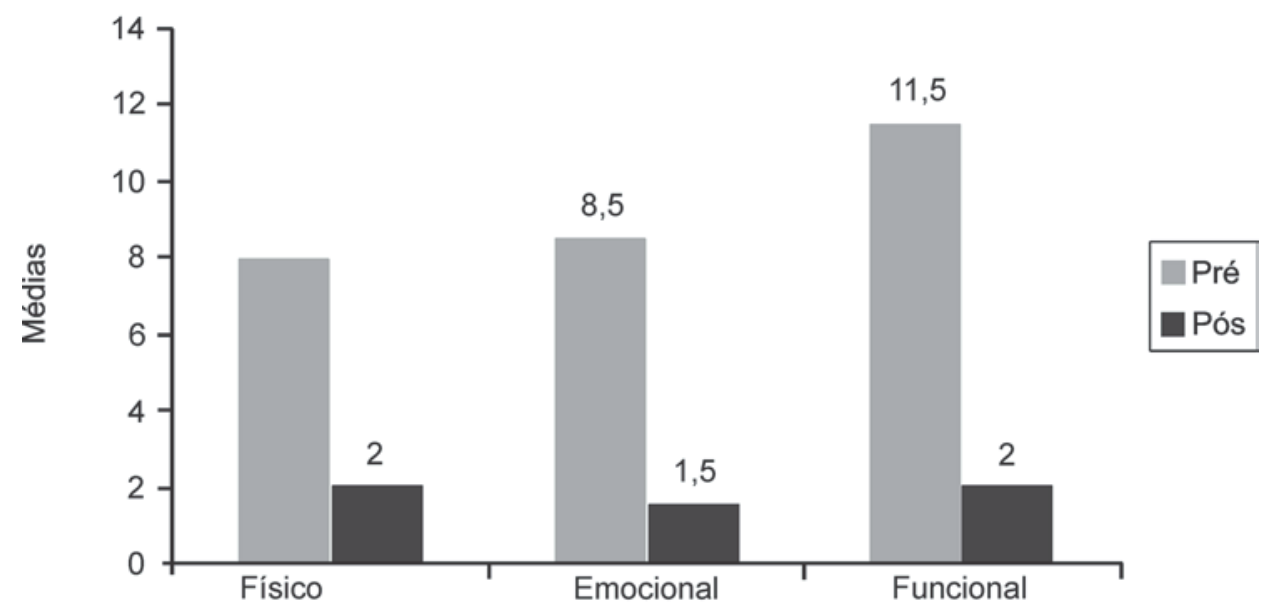

Figura 5 - Comparação das médias dos aspectos físico, funcional e emocional pré e pós reabilitação vestibular do grupo $\mathrm{A}$, utilizando o protocolo I

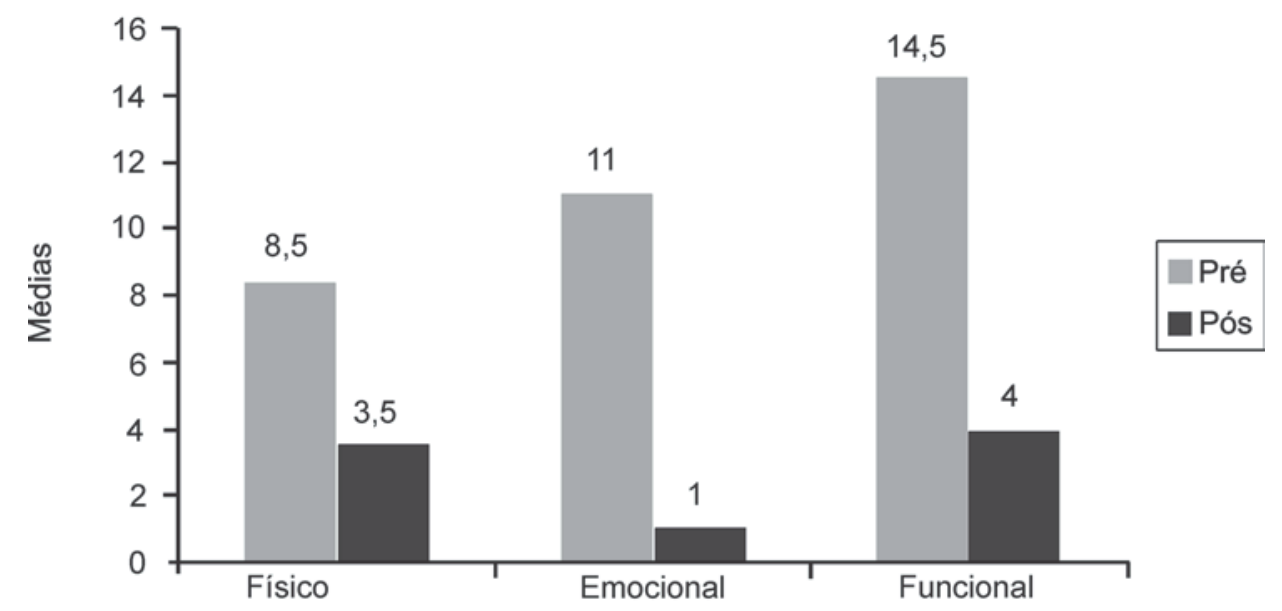

Figura 6 - Comparação das médias dos aspectos físico, funcional e emocional pré e pós reabilitação vestibular do grupo $\mathrm{B}$, utilizando o protocolo II

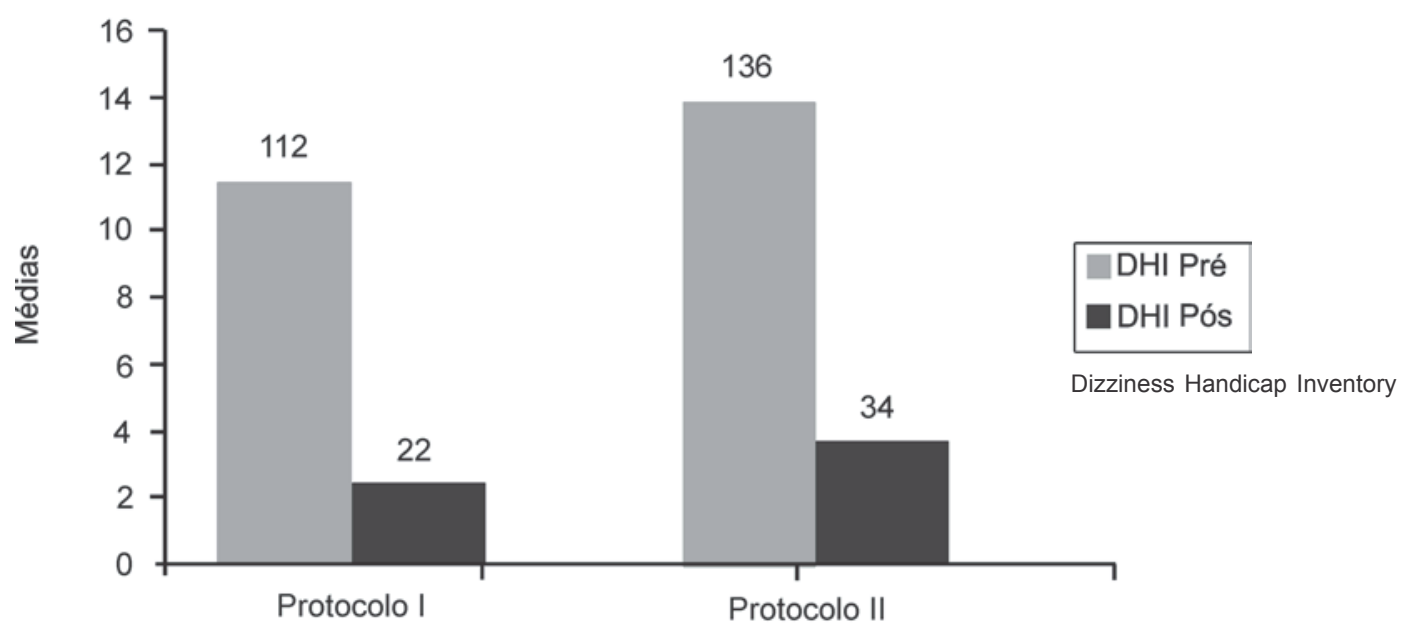

Figura 7 - Comparação entre os protocolos utilizados nos grupos A e B

Na comparação dos dois protocolos de reabilitação pré e pós-tratamento, através do teste t de Student, observou-se para o protocolo I $p=0,0231$ e para o protocolo II $p=0,060$. Dessa forma, nos pacientes submetidos à avaliação com o protocolo 1 houve uma melhora significativa, conforme Figura 7. 


\section{DISCUSSÃo}

A casuística encontra-se reduzida pelo motivo de que sete pacientes não tinham condições físicas, justificadas pela idade e pelas doenças existentes, que impossibilitavam não só o deslocamento dos mesmos para a realização do exame labiríntico como também a realização das manobras de reabilitação, com perigo de quedas e fraturas, que poderiam levar a diversos complicadores, inclusive a óbito.

A falta da dopamina no organismo, uma das aminas neurotransmissoras, resulta na degeneração neuronal. O mecanismo que ocasiona os sintomas da DP ainda é considerado complexo e pouco compreendido ${ }^{24}$.

O desequilíbrio, alterações na marcha e anormalidades posturais são freqüentes na DP. O principal sintoma decorrente à disfunção do sistema vestibular no idoso é a tontura rotatória, que pode ou não estar associada a outro tipo de tontura ${ }^{13}$.

No presente estudo, pode-se verificar pela análise da anamnese, a ocorrência e tremor, tontura, desequilíbrio à marcha, zumbido e queda. Em diversos estudos ${ }^{6,25,26}$ o tremor é a manifestação inicial da doença seguida da rigidez e bradicinesia.

O envelhecimento dos sistemas sensoriais, principalmente da visão, da propriocepção, dos receptores de pressão plantar e da função da orelha interna, em todos os níveis destes sistemas, produz uma perda neuronal que se inicia na sexta década e se acelera após os setenta anos ${ }^{27}$.

Em relação à avaliação vestibular observou-se alteração do sistema vestibular periférico em todos os idosos avaliados. A freqüência de alterações do sistema vestibular periférico é apontada em diversos estudos com idosos ${ }^{27,28}$. Quanto aos achados anormais à VENG, observou-se a prevalência de hiporreflexia, também observada como achado significativo em diversas pesquisas ${ }^{25,28}$. Já em outro estudo ${ }^{29}$, a hiperreflexia foi também observada. A redução de resposta nos testes calóricos é referida como uma das modificações do sistema vestibular em relação ao processo de envelhecimento. Diversos autores ${ }^{12,26,30}$ referem que a perda de células ciliadas das cristas ampulares e das máculas, o declínio do número de células nervosas do gânglio vestibular, a degeneração das otocônias, a diminuição do fluxo sanguíneo labiríntico, a progressiva depressão da estabilidade neural, a redução na capacidade de compen- sação dos reflexos vestíbulo-ocular (responsável em manter a visão estável durante a movimentação cefálica) e vestíbulo-espinal (responsável pela estabilização corporal) contribuem para a diminuição da velocidade dos movimentos de perseguição e para a hiporreatividade rotacional e calórica do sistema vestibular, tanto a nível periférico quanto central.

Considerando-se o DHI como uma medida de condição específica que avalia o estado de saúde de indivíduos vestibulopatas ${ }^{31}$, aplicou-se o $\mathrm{DHI}$ adaptação brasileira, pré e pós-terapia de RV. Observou-se na aplicação do protocolo I melhora significativa nos aspectos funcional e emocional no grupo A, e na aplicação do protocolo II uma melhora significativa do aspecto emocional, no grupo B. A análise das médias pré e pós-tratamento denotou melhora sintomatológica em todos os idosos.

A RV é um processo terapêutico que visa acelerar os mecanismos de compensação central por meio da plasticidade neuronal pela execução de exercícios repetitivos $^{32}$. No presente estudo, a hipótese de que os exercícios de RV podem promover a diminuição da sintomatologia vestibular é reforçada, sendo os exercícios fortes aliados no tratamento de disfunções periféricas. Na comparação dos protocolos utilizados observou-se melhora significativa no grupo $\mathrm{A}$, quando utilizado o protocolo I em relação à utilização do protocolo II, no grupo $B$. Diversos autores ${ }^{33-35}$ também aplicaram os exercícios de Cawthorne e Cooksey em seus estudos e referiram que o processo de compensação pode ser acelerado. A melhora da sintomatologia e do prognóstico clínico também foi evidenciada nos estudos ${ }^{12,36}$ utilizando o mesmo protocolo.

\section{CONCLUSÃO}

Verificou-se uma melhora dos pacientes do grupo A submetidos ao Protocolo de Cawthorne e Cooksey, principalmente para os aspectos funcional e emocional, auxiliando no processo de compensação vestibular.

Evidenciou-se que o trabalho em grupo é um forte aliado para a realização das tarefas propostas com determinação e motivação e que, apesar da casuística reduzida, a RV mostrou-se ser um recurso terapêutico efetivo e de fácil aplicação, melhorando a qualidade de vida na população estudada. 


\section{ABSTRACT}

Purpose: to check the effectiveness of vestibular rehabilitation exercises (RV) by means of an evaluation of a pre and post application of the Dizziness Handicap Inventory (DHI) questionnaire (Brazilian version). Methods: eight patients were evaluated (three female and five male), in the age group varying from 48 to 71, referred from the Paraná Association of Parkinson to the Otoneurological Laboratory of Tuiuti University of Paraná. The patients were divided in two groups and submitted to the following procedures: anamnesis, otorhinolaryngological evaluation, vestibular evaluation through vectoelectronystagmography (VENG) and an application of DHI questionnaire (Brazilian version) before and after RV, using Cawthorne and Cooksey (group A) and Herdman (group B) protocols. Results: a) regarding the otoneurological complaints referred to in the anamnesis, the prevalence of dizziness $(100.0 \%)$, trembling $(100.0 \%)$ and deviation during walking (75.0\%) were observed; $b$ ) In the vestibular exam, all patients (100.0\%) presented alterations, with the largest incidence being the deficient peripheral vestibular syndromes $(62.5 \%) ; c)$ There was significant improvement of the functional $(p=0,020470)$ and emotional $(p=0,013631)$ aspects after accomplishing the RV exercises using the Cawthorne and Cooksey protocol and of the emotional aspect $(p=0,007316)$ using Herdman protocol. Conclusion: comparing the two protocols used, a significant improvement of group A patients, submitted to the protocol of Cawthorne and Cooksey $(p=0.0231)$, was confirmed.

KEYWORDS: Vestibular Functions Tests; Parkinson Disease; Electronystagmography; Dizziness

\section{REFERÊNCIAS}

1. Guccione AA. Fisioterapia geriátrica. 2. ed. Rio de Janeiro: Guanabara Koogan; 2002. p. 180-1.

2. Jornal Gazeta do Povo. Disponível em: URL: <http://www.gazetadopovo.com.br> Acesso em: 28 mar. 2005.

3. Freitas EV, Py L, Neri AL. Tratado de geriatria e gerontologia. Rio de Janeiro: Guanabara Koogan; 2002. p. 112-27.

4. Westerberg BD, Roberson JB, Stach BA, Silverberg GD, Heit GH. The effects of posteroventral pallidotomy on balance function in patients with Parkinson's disease. Stereotact Funct Neurosurg. 2002; 79(2):75-87.

5. Menezes MS, Teive HAG. Doença de Parkinson: aspectos clínicos e cirúrgicos. Rio de Janeiro: Guanabara Koogan; 2003. p. 61-5.

6. Smeltzer SC, Bare BG. Tratado de enfermagem médico cirúrgica. 9. ed. vol. 1,4. Rio de Janeiro: Guanabara Koogan; 2002. p. 394-8.

7. Ganança FF, Castro ASO, Branco FC, Natour J. Interferência da tontura na qualidade de vida de pacientes com síndrome vestibular periférica. Rev Bras Otorrinolaringol. 2004; 70(1):94-101.

8. Nadol Junior JB, Schuknecht HF. The pathology of peripheral vestibular disorders in the elderly. Ear Nose Throat J. 1989; 68(12):930-4.

9. Zeigelboim BS, Jurkiewicz AL, Fukuda Y, Mangabeira-Albernaz PL. Alterações vestibulares em doenças degenerativas do sistema nervoso central. Pró-Fono. 2001; 13(2):263-70.

10. Smiley-Oyen AL, Cheng HY, Latt LD, Redfern MS.
Adaptation of vibration-induced postural sway in individuals with Parkinson's disease. Gait Posture. 2002; 16(2):188-97.

11. SImoceli L, Bittar RMS, Bottino MA, Bento RF. Perfil diagnóstico do idoso portador de desequilíbrio corporal: resultados preliminares. Rev Bras Otorrinolaringol. 2003; 69(6):772-7.

12. Silveira SR, Taguchi CK, Ganança FF. Análise comparativa de duas linhas de tratamento para pacientes portadores de síndromes vestibulares periféricas com idade superior a sessenta anos. Acta AWHO. 2002; 21(1):14-31.

13. Ganança MM, Caovilla HH. Como lidar com as tonturas e sintomas associados. In: Ganança MM, Munhoz MSL, Caovilla HH, Silva MLG. Estratégias terapêuticas em otoneurologia. Série Otoneurológica. São Paulo: Atheneu; 2001. p. 1-14.

14. Amá LAG, Oliveira GC. Reabilitação vestibular: nossa experiência. Rev Bras Otorrinolaringol. 1993; 60(2):113-6.

15. Rezende CR, Taguchi CK, Almeida JG, Fujita RR. Reabilitação vestibular em pacientes idosos portadores de vertigem posicional paroxístitca benigna. Rev Bras Otorrinolaringol. 2003; 69(34):34-8.

16. Brandt T, Daroff RB. Physical therapy for benign paroxysmal positioning vertigo. Arch Otolaryngol. 1980; 106:484-5.

17. Padovan I, Pansini M. New possibilities of analysis in electronystagmography. Acta Otolaryngol. 1972; 73:121-5. 18. Mangabeira-Albernaz PL, Ganança MM, Pontes PAL. Modelo operacional do aparelho vestibular. In: Mangabeira-Albernaz PL, Ganança MM. Vertigem. 2. ed. São Paulo: Moderna; 1976. p. 29-36. 
19. Jacobson GP, Newman CW. The development of the dizziness handicap inventory. Arch Otolaryngol Head Neck Surg. 1990; 116(4):424-7.

20. Castro ASO. Dizziness handicap inventory: adaptação cultural para o português brasileiro, aplicação e reprodutibilidade e comparação com os resultados à vestibulometria. [mestrado]. São Paulo (SP): Universidade Bandeirante de São Paulo; 2003.

21. Cawthorne T. The physiological basis of head exercises. J Chart Soc Physiother. 1944; 30:106-7. 22. Cooksey FS. Rehabilitation in vestibular injuries. Proc Royal Soc Méd. 1946; 39:273-8.

23. Herdman SJ. Vestibular rehabilitation. In: Baloh RW, Halmagy GM, editors. Disorders of the vestibular system. Philadelphia: Davies; 1996. p. 583-97. 24.Jones D, Godwin-Austen RB. Doença de Parkinson. In: Cash PAD. Neurologia para fisioterapeutas. São Paulo: Panamericana; 1982. p. 167-77. 25. Reichert WH, Doolittle J, McDowell FH. Vestibular dysfunction in Parkinson disease. Neurology. 1982; 32(10):1133-8.

26. Whitney SL. Tratamento do idoso com disfunção vestibular. In: Herdman SJ. Reabilitação vestibular. 2. ed. São Paulo: Manole; 2002. p. 505-28.

27. Mota PHM, Franco ES, Monteiro Pinto ES, Arieta AM. Estudo do equilíbrio no idoso por meio da electronistagmografia. Acta AWHO. Disponível em: URL:http://www.actaawho.com.br/edicao/ conteudo.asp?edi_id=6\&tpc_id=1\&con_id=19_Acesso em 06 abr. $200 \overline{5}$

28. Gushikem P. Avaliação otoneurológica em idosos com tontura [mestrado]. São Paulo (SP): Universidade Federal de São Paulo; 2001.

29. Ord RJ, Dolowitz DA. Diagnosis of early Parkinson's disease. Laryngoscope. 1969; 79(7):1275-80.

30. Hain TC, Ramaswamy TS, Hillman MA. Anatomia e fisiologia do sistema vestibular normal. In: Herdman SJ. Reabilitação vestibular. 2. ed. São Paulo: Manole; 2002. p. 3-24.

31. Gill-Body KM, Beninato M, Krebs DE. Relationship among balance impairments, functional performance and disability in people with peripheral vestibular hypofunction. Phys Ther. 2000; 80(8):748-58.

32. Cesarani A, Alpini D, Monti B, Raponi G. The treatment of acute vertigo. Neurol Sci. 2004; 25(Suppl 1):S26-30.

33. Norre ME, Beckers A. Vestibular habituations training: exercise treatment for vertigo based upon the habituation effect. Otolaryngol Head Neck Surg. 1989; 101(1):14-9.

34. Telian SA, Shepard NT, Smith-Wheelock M, Kemink JL. Habituation therapy for chronic vestibular dysfunction: preliminary results. Otolaryngol Head Neck Surg. 1990; 103(1):89-95.

35. Shepard NT, Telian SA, Smith-Wheelock M, Raj A. Vestibular and balance rehabilitation therapy. Ann Otol Rhinol Laryngol. 1993; 102(3 Pt 1):198-205.

36. O'Reilly RC, Elford B, Slater R. Effectiveness of the particle repositioning maneuver in subtypes of benign paroxysmal positional vertigo. Laryngoscope. 2000; 110(8):1385-8.
RECEBIDO EM: 09/03/2007
ACEITO EM: 09/06/2007

Endereço para correspondência:

Rua Gutenberg, $999^{\circ}$ andar

Curitiba - PR

CEP: $80420-030$

Tel/Fax: (41) 33317807

E-mail: bianca.zeigelboim@utp.br 